\title{
Estimating the Transfer Range of Plasmids Encoding Antimicrobial Resistance in a Wastewater Treatment Plant Microbial Community
}

Li, Liguan; Dechesne, Arnaud; He, Zhiming; Madsen, Jonas Stenløkke; Nesme, Joseph; Sørensen, Søren J.; Smets, Barth F.

\section{Published in:}

Environmental Science \& Technology Letters

Link to article, DOI:

10.1021/acs.estlett.8b00105

Publication date:

2018

Document Version

Peer reviewed version

Link back to DTU Orbit

Citation (APA):

Li, L., Dechesne, A., He, Z., Madsen, J. S., Nesme, J., Sørensen, S. J., \& Smets, B. F. (2018). Estimating the Transfer Range of Plasmids Encoding Antimicrobial Resistance in a Wastewater Treatment Plant Microbial Community. Environmental Science \& Technology Letters, 5(5), 260-265.

https://doi.org/10.1021/acs.estlett.8b00105

\section{General rights}

Copyright and moral rights for the publications made accessible in the public portal are retained by the authors and/or other copyright owners and it is a condition of accessing publications that users recognise and abide by the legal requirements associated with these rights.

- Users may download and print one copy of any publication from the public portal for the purpose of private study or research.

- You may not further distribute the material or use it for any profit-making activity or commercial gain

- You may freely distribute the URL identifying the publication in the public portal 
1 Title: Estimating the Transfer Range of Plasmids Encoding Antimicrobial Resistance in a

2 Wastewater Treatment Plant Microbial Community

3

4 Liguan $\mathrm{Li}^{1}$, Arnaud Dechesne ${ }^{1}$, Zhiming $\mathrm{He}^{1}$, Jonas Stenløkke Madsen², Joseph Nesme², Søren J.

5 Sørensen ${ }^{2}$, Barth F. Smets ${ }^{1, *}$

6

$7 \quad{ }^{1}$ Department of Environmental Engineering, Technical University of Denmark, 2800 Kgs. Lyngby,

8 Denmark

$9 \quad{ }^{2}$ Department of Biology, University of Copenhagen, 2100 Copenhagen, Denmark

10

$11 \quad{ }^{*}$ Corresponding author

12 Address: Department of Environmental Engineering, Technical University of Demark,

13 Bygningstorvet, Bygning 115, 2800 Kgs. Lyngby

14 Phone: +45-45252230

15 Fax: +45 45932850

16 Email: bfsm@env.dtu.dk

17

18

19

20

21

22

23

24 


\section{(} 4 7

\section{Abstract}

Wastewater treatment plants (WWTPs) have long been suggested as reservoirs and sources of antibiotic resistance genes (ARGs) in the environment. In a WWTP ecosystem, human enteric and environmental bacteria are mixed and exposed to pharmaceutical residues, potentially favoring genetic exchange and thus ARG transmission. However, the contribution of microbial communities in WWTP to ARG dissemination remains poorly understood. Here, we examined for the first time plasmid permissiveness of an activated sludge microbial community, by utilizing an established fluorescent bioreporter system. The activated sludge microbial community was challenged in standardized filter matings with one of the three multi-drug resistance plasmids (pKJK5, pB10 and RP4) harbored by Escherichia coli or Pseudomonas putida. Different donor-plasmid combinations had distinct transfer frequencies, ranging from 3 to 50 conjugation events per 100,000 cells of the WWTP microbial community. In addition, transfer was observed to a broad phylogenetic range of 13 bacterial phyla with several taxa containing potentially pathogenic species. Preferential transfer to taxa belonging to the predicted evolutionary host range of the plasmids was not observed. Overall, the ARG dissemination potential uncovered in WWTP communities calls for a thorough risk assessment of ARG transmission across the wastewater system, before identifying possible mitigation strategies. (1) 5 6 8 
Wastewater treatment plants (WWTPs), at the interface between hospital/residential sewage and recipient surface water, have been proposed as overlooked reservoirs of antibiotic resistance genes (ARGs). ${ }^{1-3}$ Indeed, there, the microbiomes indigenous to WWTP are intensely mixed with

53 54

\section{4} microbiomes of human enteric origin, in the presence of pharmaceutical residues and other selective agents, potentially stimulating the transfer of ARGs from pathogens and commensals to environmental bacteria. Among the gene transfer processes (e.g., transformation, transduction and conjugation), plasmid-mediated conjugation is characterized by its efficiency, even across distantly related taxa for broad host range plasmids. Therefore, the transfer of ARGs is facilitated by their frequent location on plasmids. ${ }^{4-6}$ Several studies have provided evidence that WWTP microbiomes can contain significant amount of plasmids encoding multi-drug resistance. ${ }^{7-9}$ Environmental bacteria receiving these plasmid-borne ARGs can persist in the receiving environments, facilitating their dissemination. ${ }^{10,11}$ Considering the global public health threat posed by antimicrobial resistance and the obvious load from human waste collected and transported through sewage, it is crucial to evaluate the potential contribution of WWTP to plasmid mediated ARG dissemination.

In order to understand the fate of ARG-carrying plasmids in WWTP ecosystems, it is necessary to disentangle the roles of plasmid type, donor strain, and resident microbial community in shaping the plasmid transfer host ranges. The plasmid permissiveness assay, as originally introduced by Musovic et al, ${ }^{12}$ provides a suitable platform to address this question. Combining a fluorescent reporter based plasmid detection assay with fluorescence-activated cell sorting (FACS) and 16S rRNA gene amplicon sequencing of transconjugant cells, it enables quantification and identification of the community fraction that receives the tested plasmid upon challenging this community with a plasmid donor strain. ${ }^{13-15}$ Using this approach, extremely broad transfer host ranges of IncP-1 
conjugative plasmid pKJK5 have been detected in microbial communities from agricultural soil, ${ }^{13,14}$ as well as from the inlet and outlet of WWTPs. ${ }^{15}$ Yet, the permissiveness of WWTP microbial communities for typical and relevant IncP-1 plasmids of different subgroups has not been examined. It has been argued - mainly based on metagenomic observations - that the high species diversity and cellular density of WWTP microbial communities creates a locale favoring horizontal gene transfer. ${ }^{8,16}$ Predicting the range of plasmid-mediated genetic exchange at the community level has so far not been possible; host ranges inferred from bioinformatic analyses or traditional assays have been skewed toward only identifying evolutionary host taxa with preexisting genomic homogeneity or examining a limited number of well-studied model strains. ${ }^{17-20}$ We believe that direct confirmation and quantification of this exchange is, however, necessary and possible via plasmid permissiveness assays. ${ }^{13-15}$ By quantifying and identifying the permissive fraction, one can evaluate plasmid transfer potential as an essential community property and examine abiotic/biotic factors (e.g., environmental conditions, plasmid/donor type and recipient community) that shape permissiveness profiles, which together will help understand plasmid-mediated ARG spread.

8 Here, we report on the first permissiveness estimates of a WWTP microbial community towards several typical conjugative plasmids, and the first exploration of association between plasmid transfer and evolutionary host ranges. A WWTP community was challenged with three ARGcarrying plasmids from different subgroups in the incompatibility group IncP-1 (pKJK5, pB10 and RP4) ${ }^{17}$ using either the prototypic member of Enterobacteriaceae - Escherichia coli or typical environmental bacterium - Pseudomonas putida as donor strains. Distinct transfer potentials were observed with the highest realized in E. coli (pKJK5) (50 conjugation events per 100,000 recipient cells). The transfer host ranges covered 13 phyla across the different donor-plasmid combinations; but no preferred transfer was observed to taxa predicted to belonging to the evolutionary host range 

111 filter mating assays.

\section{Material and methods} WWTP recipient cell number (CE/R), as per established procedures. ${ }^{13,14,23}$

of the plasmids. It is noteworthy that plasmid acquisition was observed in several taxa with potentially pathogenic species. Overall, the wide transfer potential of plasmids experimentally revealed in this study confirms the importance of WWTP as a unique locale for plasmid mediated ARGs exchange between enteric and environmental bacteria.

\section{Donor strain and WWTP recipient community}

E. coli MG1655 and P. putida KT2440 (both chromosomally tagged by lacI ${ }^{q}$-Plpp-mCherry)

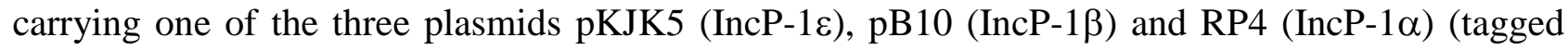
with Plac-gfp), were used as donors (each combination group will be referred to as donor (plasmid), e.g., E. coli (pKJK5)) (Table S1). The donor strains were grown overnight in LB prepared as described previously. ${ }^{13,14}$ Recipient community was phase-isolated activated sludge from a municipal WWTP (Mølleåværket, Lyngby-Taarbæk, DK). Briefly, bacteria were recovered by washing, sonication and settling. Cell numbers were adjusted to approx. $3.0 \times 10^{7}$ cells per ml for

\section{Solid surface filter mating assay}

Cell suspensions of donor strain and WWTP recipient community were mixed at 1:1 cell ratio and immediately filtered onto $0.2 \mu \mathrm{m}$ Cyclopore membranes. ${ }^{21}$ Filters were placed on a agar-solidified synthetic wastewater medium..$^{22}$ After incubation (48 hours at $25^{\circ} \mathrm{C}$ ) and GFP maturation (48 hours at $4^{\circ} \mathrm{C}$ ), transfer events were detected by epifluorescence microscopy and transfer frequency was quantified as the ratio of conjugation events (GFP-positive cells or microcolonies) to the original 


\section{Sorting and sequencing}

122 For each mating condition, cells from triplicate filters were combined in $0.9 \% \mathrm{NaCl}$ solution and 123 detached by vortexing. Transconjugants and recipients were sorted using FACS by adjusting gating 124 of bacterial size (forward scatter), green fluorescence, and red fluorescence as described earlier. ${ }^{13,14}$ 125 Sorted cells were subject to DNA extraction using GenePurgeDirect ${ }^{\mathrm{TM}}$ agent (NimaGen, NL). 16S 126 rRNA gene fragments were amplified by the primer set $341 \mathrm{~F}$ and $806 \mathrm{R},{ }^{15}$ and subjected to paired127 end sequencing on Illumina MiSeq platform.

\section{Sequence analysis}

130 The forward reads of the 16S rRNA gene amplicon sequencing were analyzed using the DADA2 131 pipeline to infer exact sequence variants (ESV) (Table S2). ${ }^{24,25}$ As estimating ESV-specific 132 permissiveness is complicated by the (potential) growth of both transconjugants and recipients 133 during mating incubation, we calculate apparent permissiveness (AP). It is defined as the ratio of 134 the relative abundance of an ESV in the transconjugant pool and in the corresponding recipient 135 community. ${ }^{14}$ AP thus accounts for the fact that the abundance for an ESV in the transconjugant 136 pool is partly dependent on their abundance in the recipient community. Phylogenetic relatedness 137 between donor and transconjugant was calculated by DistanceMatrix in R package DECIPHER ${ }^{26}$ 138 and its correlation with AP values was calculated with Spearman correlation coefficient. 139 Phylogenetic conservation of AP values was analyzed by calculating their phylogenetic signal in 140 corresponding ESVs by multiPhylosignal in R package picante. ${ }^{27}$ Plasmid transfer host range and 141 evolutionary host range (i.e., hosts that have carried the plasmid during evolutionary time long 142 enough to leave detectable sequence traits), were compared based on previous genomic analysis. ${ }^{17}$ 143 Occurrence of these evolutionary hosts in transconjugant pools was evaluated by t-test of both 144 relative abundance and AP value. All sequences were deposited in NCBI under SRA accession 
number SRP133153. Method details including experimental setups and statistical analyses are provided in Supporting Information.

\section{Results and Discussion}

Transfer frequencies across donor-plasmid combinations

150 Transfer frequencies in the WWTP microbial community ranged from $3.39 \times 10^{-5}$ to $5.05 \times 10^{-4}$ 151 CE/R (i.e., from 3 to 50 conjugation events per 100,000 recipient cells) across donor-plasmid 152 combinations (Figure 1); comparable transfer frequencies have been measured in soil microbial 153 communities $\left(6.8 \times 10^{-5} \mathrm{CE} / \mathrm{R} \text { of E. coli (pKJK5) and } 1.0 \times 10^{-4} \mathrm{CE} / \mathrm{R} \text { of } P \text {. putida (RP4) }\right)^{13,28}$ All 154 three plasmids transferred at higher frequency from E. coli compared to $P$. putida with the highest 155 transfer frequency observed with E. coli (pKJK5). Comparison among transfer frequencies of the 156 three plasmids carried by the same host showed that pKJK5>pB10>RP4 in E. coli and

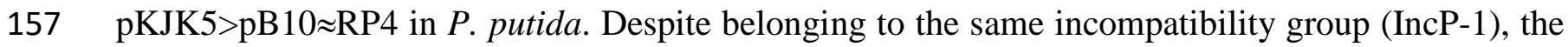
158 three plasmids present genetic divergence in their transfer and regulatory regions, ${ }^{17,29}$ which might 159 explain the difference in observed conjugation behavior.

\section{Transfer host ranges for different donor-plasmid combinations}

Recipient communities were distinct from transconjugant pools (NMDS, ANOSIM P-value<0.01).

163 While post filter-mating recipient pools were distinct from the raw AS communities, reasonable 164 diversity was retained: the Shannon diversity index decreased slightly from 5.3 to 4.7. And 165 notwithstanding the presence of a shared core (see below) the four transconjugant pools were 166 distinct from each other (Figure 2). As expected, recipient pools were more diverse than 167 transconjugant pools (Shannon diversity $=4.2-4.6$; unique ESVs $=229-360$ vs Shannon diversity $=$ 168 1.3-3.2; unique ESVs = 73-126). Interestingly, distances within transconjugant pools and recipient 
communities of E. coli/P . putida (pKJK5) were similar (Bray-Curtis distance within transconjugant pools vs within recipient pools $=0.55$ vs 0.63$)$. However, the three transconjugant pools of $E$. coli (pKJK5/pB10/RP4) were clearly distinct from each other, even though their recipient communities were close (Bray-Curtis distance $=0.43-0.57$ vs 0.23-0.26). Hence, a plasmid type might shape transconjugant pool composition more than a plasmid donor.

The transconjugants across all donor-plasmid combinations comprised 308 distinct ESVs distributed over 13 phyla (Figure 2; Figure 3). While all transconjugant pools were dominated by genera from the Gammaproteobacteria class including Escherichia/Shigella, Pseudomonas and Acinetobacter, a few other Gram-negative (Chloroflexi, Acidobacteria and Bacteroidetes) and Gram-positive (Actinobacteria and Firmicutes) taxa were also noted. Overall, plasmid transfer was observed in 34-59\% of the families present in the recipient community. Thirteen permissive genera were shared across all donor-plasmid combinations, representing $>80 \%$ of each transconjugant pool. These core permissive taxa were mainly composed by Enterobacteriaceae and Pseudomonadaceae. These two lineages were also detected in transconjugant pools when permissiveness of inlet and outlet of the same WWTP was examined, ${ }^{15}$ indicating their possible transmission from sewage to the environment. The frequent occurrence of Acinetobacter, Aeromonas and Streptococcus in the transconjugant pools highlights the possibility of ARG transmission to (opportunistic) pathogens. The high frequency and broad range of plasmid transfer to the examined WWTP community under the standardized experimental conditions, in the absence of selective pressure, suggests significant ability of ARG spread under actual WWTP conditions of intense microbial interaction ${ }^{30}$ and the presence of residual antibiotics and other relevant co-selective stressors. ${ }^{31}$

\section{Heterogeneous apparent permissiveness profiles}


193 The relative abundance profile of community members in transconjugant pools did not agree with 194 their abundance in the recipient pools, indicating that capability in receiving plasmids varied among 195 taxa (Figure S1): a few taxa with low abundance in the recipient communities were highly enriched 196 in the transconjugant pools across all donor-plasmid combinations (e.g., Escherichia/Shigella $<1 \%$ 197 in recipient pools and $>40 \%$ in all transconjugant pools; Shimwellia was $<0.1 \%$ in recipient pools 198 but $>2 \%$ in transconjugant pools with both E. coli (pKJK5) and $P$. putida (pKJK5) groups). On the 199 contrary, some highly abundant taxa were poorly represented in the transconjugant pools indicating 200 their poor permissiveness (e.g., Acinetobacteria $>40 \%$ in recipient but $<0.1 \%$ in the transconjugant 201 pool with E. coli (pB10) group). In several abundant taxa, no plasmid transfer was detected (e.g., 202 Flavobacterium at 8-10\% in recipient communities while absent in transconjugant pool of E. coli 203 (pB10)).

Phylogenetic relatedness between recipient and donor did not explain the composition of the transconjugant pools for the three examined IncP-1 plasmids (Figure S1 and S2). Certainly, high intra-generic transfer was observed: from donor E. coli to Escherichia/Shigella (AP up to 704.1) 208 and from donor P. putida to Pseudomonas (AP up to 294.2). However, transfer to distant 209 phylogenetic groups, even across phylum borders, was equally observed, e.g., E. coli (pKJK5) transferred at high frequency to Pseudobacteroides (Firmicutes) (AP up to 448.1) and Gardnerella 211 (Actinobacteria) (AP up to 429.6). Hence, the AP profile did not correlate with the phylogenetic 212 distance between recipient and donor (Spearman correlation, P-value $=0.10 \sim 0.93$ ). Within a single 213 permissive genus, APs could be similar in magnitude or vary greatly: e.g., with E. coli (pB10) and 214 E. coli (RP4), APs of Staphylococcus ESVs were within one order of magnitude; with E. coli 215 (pKJK5), APs of Acinetobacter and Pseudomonas ESVs each ranged over three orders of 216 magnitude. Such varying response at the ESV level indicates that AP is not significantly 
phylogenetically conserved (phylogenetic signal, P-value $=0.61 \sim 0.98$ ). Therefore, for the three IncP-1 plasmids, extrapolating permissiveness of a bacterial group to other phylogenetically similar groups in the WWTP community would not be valid. Future studies, including more plasmid groups, and especially plasmids with assumed narrow-host-range groups (e.g., IncF and IncI), will reveal the generality of this conclusion.

\section{Comparing transfer host range to predicted evolutionary host range}

While plasmid transfer host range can be inferred from experimental permissiveness assays, it is not clear how this range relates with a plasmid's long-term maintenance as plasmid acquisition is only the very first step of a possible long-term plasmid-host association. Since long-term adaptation between plasmid and host is achieved through genomic homogenization and subsequent cost amelioration, a plasmid's evolutionary host range can be inferred from genomic comparisons between bacterial chromosomes and plasmids (backbone). ${ }^{17,18}$ For example, for the three examined plasmids, pKJK5 was predicted to have been evolutionarily present in the genera Bordetella, Dechloromonas and Pseudomonas, pB10 in Ralstonia and Variovorax, and RP4 in Ralstonia, Slackia and Pseudomonas. ${ }^{17}$ These predicted evolutionary hosts might have more potential in taking up the plasmid and expressing its genes because of the preexisting genomic homogeneity. However, we did not detect such enrichment for members of the predicted evolutionary host range of the three plasmids in their corresponding transconjugant pools (Table S3). Among the six genera belonging to the predicted evolutionary host range of the three plasmids, only Pseudomonas $(0.25 \% \sim 17.60 \%$ 237 with AP 0.5-294.2 across all groups) and Dechloromonas (0.14\% with AP 2.3 in E. coli (pKJK5)) were detected in transconjugant pools. Even at higher taxonomical levels, there was little indication of enrichment of evolutionary host taxa in the transconjugant pools. For example, Burkholderiaceae 240 (family) predicted as evolutionary host taxon of pB10, were not observed in the pB10 
241 transconjugant pool; Burkholderiales (order) were observed in the pKJK5 transonjugal pools with E. 242 coli as donor but below 1\% with AP ranging from 0.2-262.6; Gram-positive Actinobacteria (class) 243 predicted evolutionary hosts of RP4, were minor fractions of the RP4 transconjugant pools $(<4 \%$ 244 with AP ranging 0.2-429.6). Hence, evolutionary host range predicted from genomic analysis does 245 not seem to reflect extant plasmid transfer host rang in WWTP microbial communities.

247 In this study, the dissemination potential of ARGs in environmental communities was highlighted 248 by the high transfer frequency (up to 50 conjugation events per 100,000 recipient cells) and the 249 broad phylogenetic transfer range (covering 13 phyla) of the three ARG-carrying plasmids in a 250 WWTP microbial community. Taxa belonging to a plasmid's predicted evolutionary host range do 251 not necessarily exhibit high permissiveness. The plasmid permissiveness assay as adapted here for 252 WWTP communities provides a quantitative assessment of a community property that is essential, 253 but not sufficient, to describe, and ultimately predict the fate of plasmids in the environment. Indeed, 254 the potential for plasmid uptake, as measured here, is not realized in situ in WWTP systems, and 255 extrapolation to real environments will require additional experiments to identify the role of the 256 environment, including conditions of (sub)inhibitory selective or co-selective pressure.

258 Acknowledgements

259 This work was supported by the Joint Programming Initiative-Antimicrobial Resistance (JPI-AMR; 260 DARWIN project \#7044-00004B) to BFS; and the H.C. Ørsted Postdoc programme co-funded by 261 Marie Skłodowska-Curie Actions to LL.

\section{Conflict of interest}

264 The authors declare no competing financial interest. 


\section{Supporting Information}

267 Supplementary methods of plasmid donor strain and recipient microbial community, solid surface filter mating assay, sorting and sequencing (sequence analysis); supplementary figures of relative abundance of genera across samples, AP profile of ESVs; supplementary tables of donor strains and plasmids, information of sequences, relative abundance of predicted evolutionary taxa.

\section{References}

(1) Baquero, F.; Martínez, J. L.; Cantón, R. Antibiotics and antibiotic resistance in water

(4) Crofts, T. S.; Gasparrini, A. J.; Dantas, G. Next-generation approaches to understand and combat the antibiotic resistome. Nat. Rev. Microbiol. 2017, 15 (7), 422-434, DOI: 10.1038/nrmicro.2017.28.

(5) Sun, J.; Yang, R.-S.; Zhang, Q.; Feng, Y.; Fang, L.-X.; Xia, J.; Li, L.; Lv, X.-Y.; Duan, J.-H.; Liao, X.-P.; et al. Co-transfer of bla $a_{\mathrm{NDM}-5}$ and $m c r-1$ by an IncX3-X4 hybrid plasmid in Escherichia coli. Nat. Microbiol. 2016, 1 (September), 16176, DOI: 10.1038/nmicrobiol.2016.176.

(6) Liu, Y. Y.; Wang, Y.; Walsh, T. R.; Yi, L. X.; Zhang, R.; Spencer, J.; Doi, Y.; Tian, G.; Dong, B.; Huang, X.; et al. Emergence of plasmid-mediated colistin resistance mechanism MCR-1 in animals and human beings in China: A microbiological and molecular biological study. Lancet Infect. Dis. 2016, 16 (2), 161-168, DOI: 10.1016/S1473-3099(15)00424-7. 
(7) Schlüter, A.; Szczepanowski, R.; Pühler, A.; Top, E. M. Genomics of IncP-1 antibiotic resistance plasmids isolated from wastewater treatment plants provides evidence for a widely accessible drug resistance gene pool. FEMS Microbiol. Rev. 2007, 31 (4), 449-477, DOI: 10.1111/j.1574-6976.2007.00074.X.

(8) Zhang, T.; Zhang, X.-X.; Ye, L. Plasmid metagenome reveals high levels of antibiotic 300

(9) Guo, J.; Li, J.; Chen, H.; Bond, P. L.; Yuan, Z. Metagenomic analysis reveals wastewater 303

(10) Suhartono, S.; Savin, M.; Gbur, E. E. Genetic redundancy and persistence of plasmid306

(11) Czekalski, N.; Gascón Díez, E.; Bürgmann, H. Wastewater as a point source of antibiotic309

(12) Musovic, S.; Dechesne, A.; Sørensen, J.; Smets, B. F. Novel assay to assess permissiveness

(14) Klümper, U.; Dechesne, A.; Riber, L.; Brandt, K. K.; Gülay, A.; Sørensen, S. J.; Smets, B. F. Metal stressors consistently modulate bacterial conjugal plasmid uptake potential in a phylogenetically conserved manner. ISME J. 2017, 11, 152-165, DOI: 10.1038/ismej.2016.98.

(15) Jacquiod, S.; Brejnrod, A.; Morberg, S. M.; Abu Al-Soud, W.; Sørensen, S. J.; Riber, L. Deciphering conjugative plasmid permissiveness in wastewater microbiomes. Mol. Ecol. 2017, 26 (13), 3556-3571, DOI: 10.1111/mec.14138.

(16) Li, A.-D.; Li, L.-G.; Zhang, T. Exploring antibiotic resistance genes and metal resistance genes in plasmid metagenomes from wastewater treatment plants. Front. Microbiol. 2015, 6 (September) DOI: 10.3389/fmicb.2015.01025. 
(17) Norberg, P.; Bergström, M.; Jethava, V.; Dubhashi, D.; Hermansson, M. The IncP-1 plasmid backbone adapts to different host bacterial species and evolves through homologous recombination. Nat. Commun. 2011, 2, 268, DOI: 10.1038/ncomms1267.

(18) Suzuki, H.; Yano, H.; Brown, C. J.; Top, E. M. Predicting plasmid promiscuity based on genomic signature. J. Bacteriol. 2010, 192 (22), 6045-6055, DOI: 10.1128/JB.00277-10.

(19) Hall, J. P. J.; Wood, A. J.; Harrison, E.; Brockhurst, M. A. Source-sink plasmid transfer dynamics maintain gene mobility in soil bacterial communities. Proc. Natl. Acad. Sci. 2016, 113 (29), 8260-8265, DOI: 10.1073/pnas.1600974113.

(20) Yano, H.; Rogers, L. M.; Knox, M. G.; Heuer, H.; Smalla, K.; Brown, C. J.; Top, E. M. Host range diversification within the IncP-1 plasmid group. Microbiology 2013, 159, 2303-2315, DOI: $10.1099 /$ mic.0.068387-0.

(21) Klümper, U.; Dechesne, A.; Smets, B. F. Protocol for evaluating the permissiveness of bacterial communities toward conjugal plasmids by quantification and isolation of transconjugants. In Hydrocarbon and Lipid Microbiology Protocols: Genetic, Genomic and System Analyses of Communities; McGenity, T. J., Timmis, K. N., Balbina, N., Eds.; Springer Berlin Heidelberg, 2014; pp 275-288.

(22) Test No. 303: Simulation Test - Aerobic Sewage Treatment. In OECD Guidelines for the Testing of Chemicals, Section 3; OECD Publishing, 2013; p 50.

(23) Klümper, U.; Droumpali, A.; Dechesne, A.; Smets, B. F. Novel assay to measure the plasmid mobilizing potential of mixed microbial communities. Front. Microbiol. 2014, 5 (December), 730, DOI: 10.3389/fmicb.2014.00730.

(24) Callahan, B. J.; McMurdie, P. J.; Rosen, M. J.; Han, A. W.; Johnson, A. J. A.; Holmes, S. P. DADA2: High-resolution sample inference from Illumina amplicon data. Nat. Methods 2016, 13 (7), 581-583, DOI: 10.1038/nmeth.3869.

(25) Callahan, B. J.; McMurdie, P. J.; Holmes, S. P. Exact sequence variants should replace operational taxonomic units in marker-gene data analysis. ISME J. 2017 DOI: 10.1038/ismej.2017.119.

(26) Wright, E. S. DECIPHER: harnessing local sequence context to improve protein multiple sequence alignment. BMC Bioinformatics 2015, 16 (1), 322, DOI: 10.1186/s12859-0150749-z.

(27) Kembel, S. W.; Cowan, P. D.; Helmus, M. R.; Cornwell, W. K.; Morlon, H.; Ackerly, D. D.; Blomberg, S. P.; Webb, C. O. Picante: R tools for integrating phylogenies and ecology. 
(28) Musovic, S.; Klümper, U.; Dechesne, A.; Magid, J.; Smets, B. F. Long-term manure exposure increases soil bacterial community potential for plasmid uptake. Environ. Microbiol. Rep. 2014, 6 (2), 125-130, DOI: 10.1111/1758-2229.12138.

(29) Sen, D.; Brown, C. J.; Top, E. M.; Sullivan, J. Inferring the evolutionary history of IncP-1 plasmids despite incongruence among backbone gene trees. Mol. Biol. Evol. 2013, 30 (1), 154-166, DOI: 10.1093/molbev/mss210.

(30) Zhang, T.; Shao, M.-F.; Ye, L. 454 Pyrosequencing reveals bacterial diversity of activated sludge from 14 sewage treatment plants. ISME J. 2011, 6, 1137-1147, DOI: 10.1038/ismej.2011.188.

(31) Seiler, C.; Berendonk, T. Heavy metal driven co-selection of antibiotic resistance in soil and water bodies impacted by agriculture and aquaculture. Front. Microbiol. 2012, 3, 399, DOI: 10.3389/fmicb.2012.00399. 
389 Figure 1. Transfer frequencies (CE/R: the ratio of conjugation events (CE) to the original WWTP 390 recipient cell number (R)) from two donors (E. coli and P. putida) carrying one of three plasmids 391 (pKJK5, RP4 and pB10) to an activated sludge microbial community. Error bar indicates 95\% 392 confidence interval of three replicates.

394 Figure 2. Diversity and phylogenetic composition of transconjugant and recipient communities. (A) 395 and (B): Shannon index and NMDS (the same color scheme was applied in the two panels; for each 396 donor-plasmid combination (circle dots), dark color indicates transconjugant pools and light color 397 (within ellipse) indicates recipient pools; triangle dots indicate WWTP microbial communities. (C) 398 and (D): phylogenetic composition at phylum level and relative abundance of phyla except Gamma399 and Alpha-proteobacteria in transconjugant pools. (E) and (F): top 20 abundant orders and genera in 400 the transconjugant pools.

401

402 Figure 3. Composition of the transconjugant pools across four donor-plasmid combinations. (A) 403 and (B): phylogenetic tree showing the relative abundance of ESVs detected with E. coli 404 (pKJK5/RP4/PB10) and E. coli/P. putida (pKJK5) as plasmid donor. Background colors indicate 405 the 6 most abundant classes, and branch colors indicate the 13 core genera across all transconjugant 406 pools (refer to panel (E)). (C), (D) and (E): Venn diagrams at the genus level of the transconjugant 407 pools of E. coli (pKJK5/RP4/PB10), E. coli/P. putida (pKJK5) and all groups. 
Figure 1. Transfer frequencies (CE/R: the ratio of conjugation events (CE) to the original WWTP 425 recipient cell number (R)) from two donors (E. coli and $P$. putida) carrying one of three plasmids

426 (pKJK5, RP4 and pB10) to an activated sludge microbial community. Error bar indicates 95\%

427 confidence interval of three replicates. $0 \mathrm{e}+00$

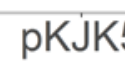

E. coli

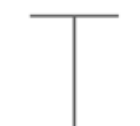
E. coli
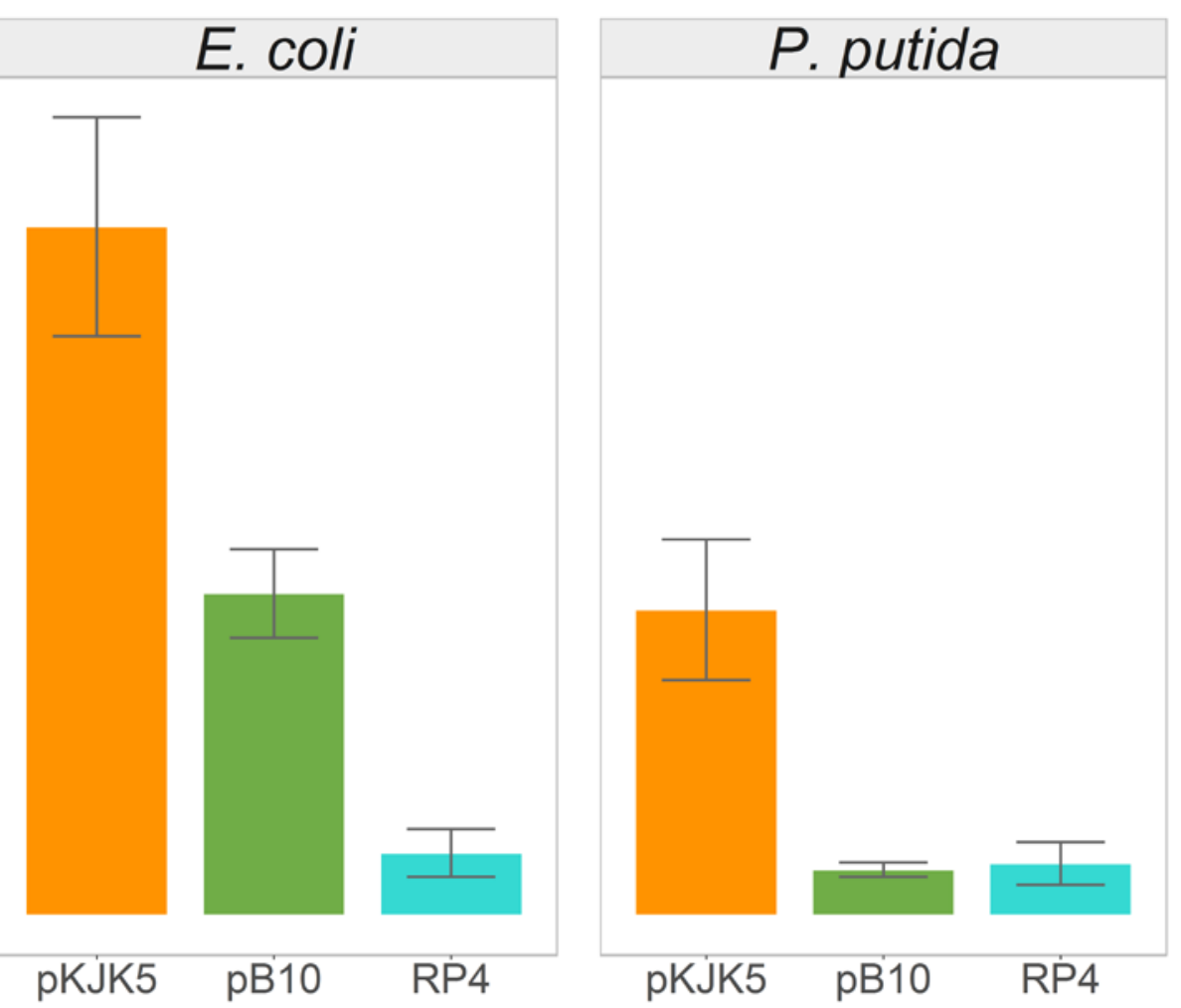

plasmid

428

429

430

431

432

433

434 
A

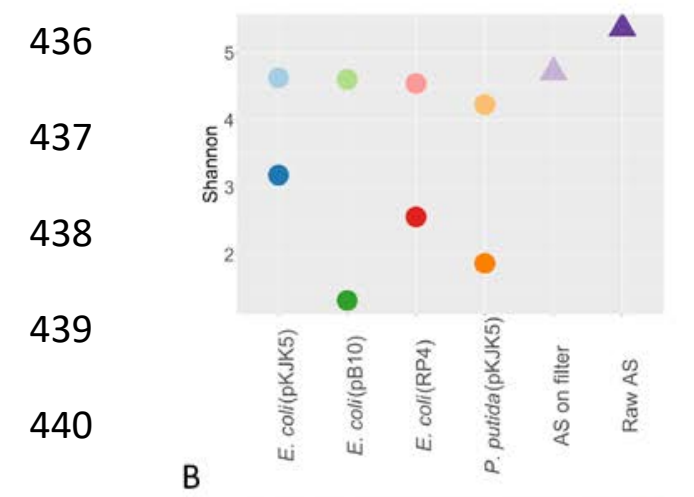

441

442

443

444

445

446

447

448

449

450

451

452

453

454 the transconjugant pools.
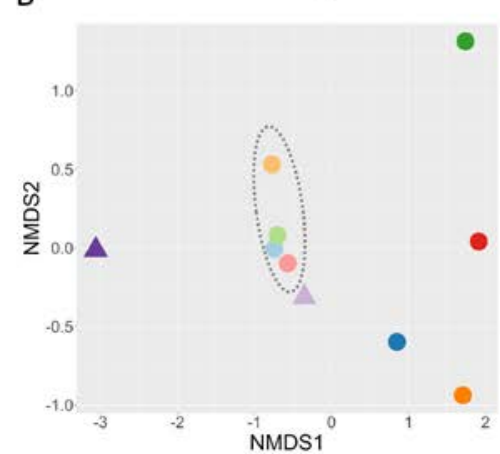

C

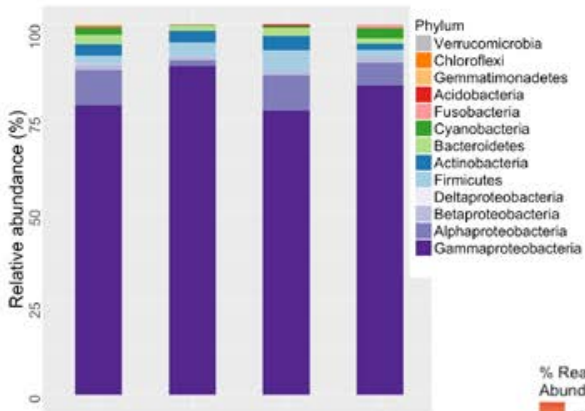

D

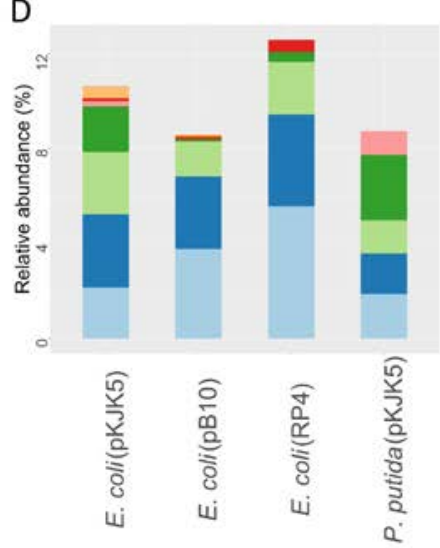

E
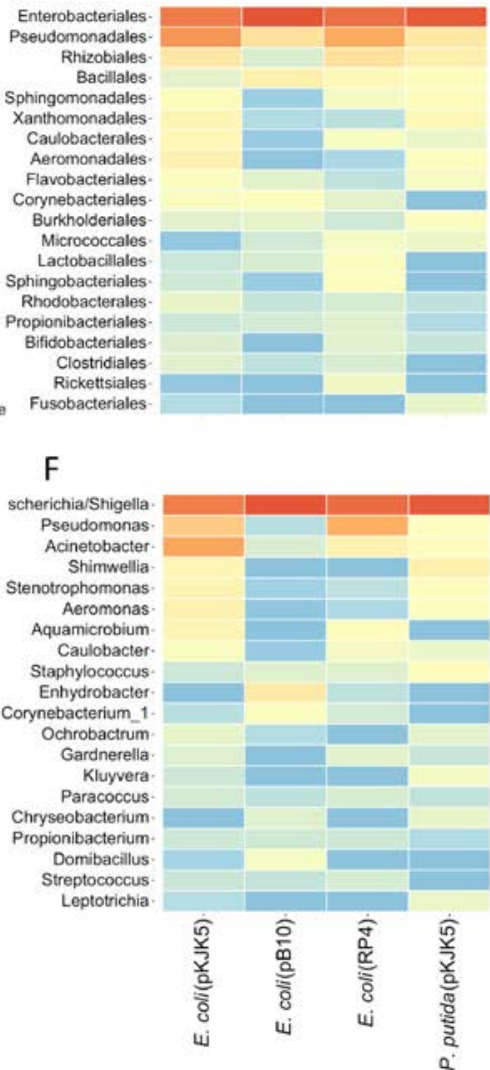

Figure 2. Diversity and phylogenetic composition of transconjugant and recipient communities. (A) and (B): Shannon index and NMDS (the same color scheme was applied in the two panels; for each donor-plasmid combination (circle dots), dark color indicates transconjugant pools and light color (within ellipse) indicates recipient pools; triangle dots indicate WWTP microbial communities. (C) and (D): phylogenetic composition at phylum level and relative abundance of phyla except Gammaand Alpha-proteobacteria in transconjugant pools. (E) and (F): top 20 abundant orders and genera in 455

456 
A

460

461

462

463

464

465

466

467

468

469

470

471

472

473

474

475

476

477

478

479

480

481

482

483
C

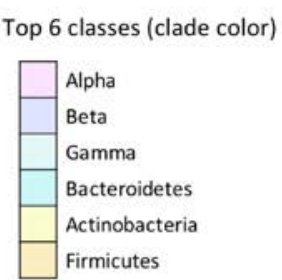

D

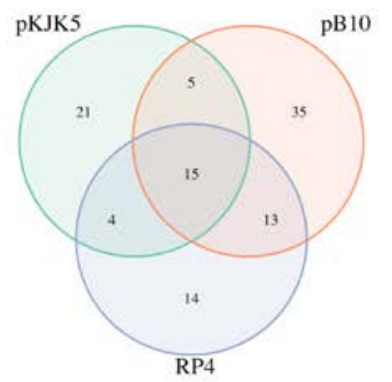

Relative abundance(log transform) (heatmap color)
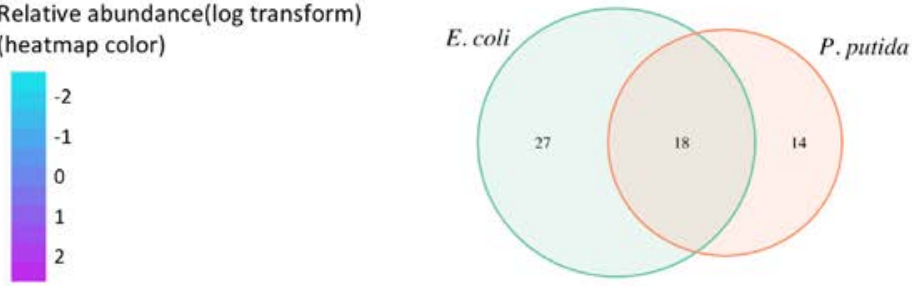

13 core genera in all transconjugants (branch color)
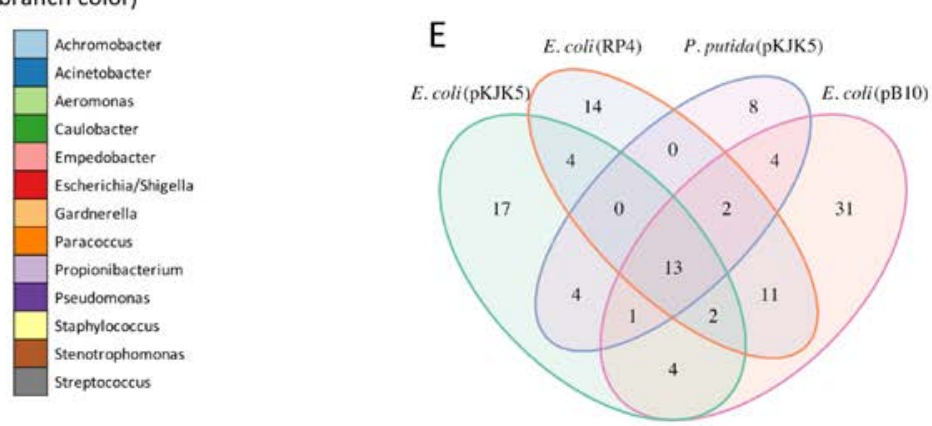

ter

Figure 3. Composition of the transconjugant pools across four donor-plasmid combinations. (A) and (B): phylogenetic tree showing the relative abundance of ESVs detected with E. coli (pKJK5/RP4/PB10) and E. coli/P. putida (pKJK5) as plasmid donor. Background colors indicate the 6 most abundant classes, and branch colors indicate the 13 core genera across all transconjugant pools (refer to panel (E)). (C), (D) and (E): Venn diagrams at the genus level of the transconjugant pools of E. coli (pKJK5/RP4/PB10), E. coli/P. putida (pKJK5) and all groups.

$$
\text { B }
$$

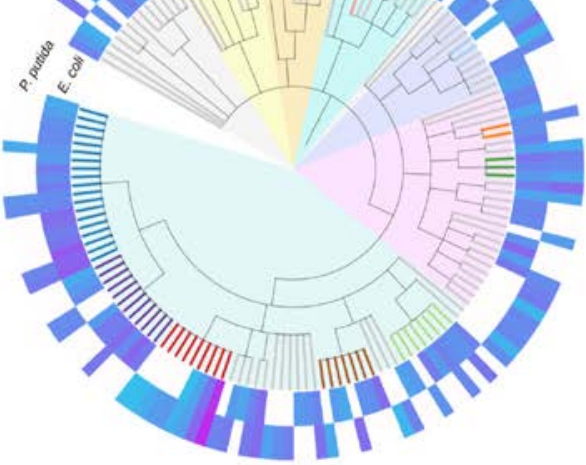




\section{For Table of Contents Use Only}

485

486 Title: Estimating the Transfer Range of Plasmids Encoding Antimicrobial Resistance in a

487 Wastewater Treatment Plant Microbial Community

488

489 Liguan Li, Arnaud Dechesne, Zhiming He, Jonas Stenløkke Madsen, Joseph Nesme, Søren J.

490 Sørensen, Barth F. Smets

491

492

493

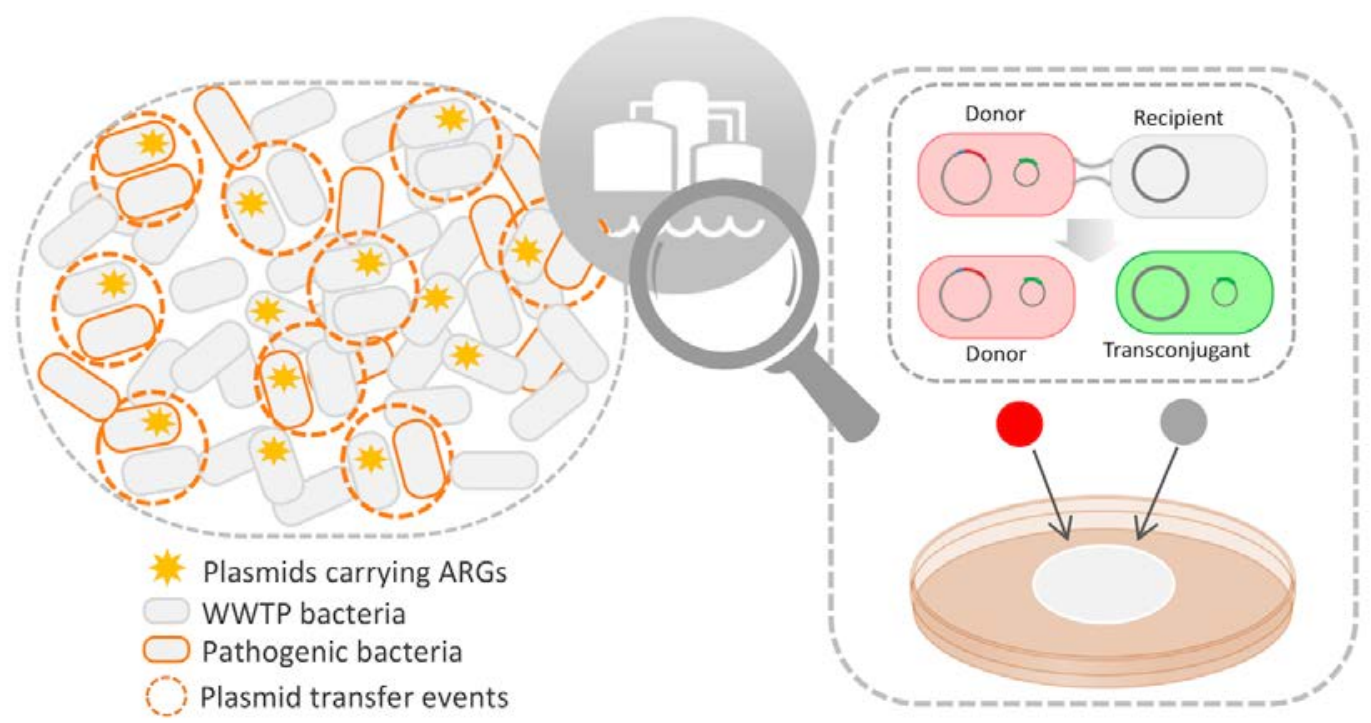

